\title{
EDITORIAL
}

\section{The Limbus}

The articles in this issue and in some of the next are from the Cambridge Ophthalmological Symposium in 1988, the subject of which was 'The Limbus'.

All junction zones are interesting whether they be simply the regions where mucous membranes meet skin or complex associations that exist between for instance the anterior and posterior pituitary and in the separations of tissues of neural origin from those of somatic origin. The limbus is just as extraordinary as any of these. This is the area in which the opaque sclera is transformed into the transparent cornea. To do so its collagenous structure has to change, the size of its fibres have to change, it has to develop specialised tissue to ensure that the cornea is kept dehydrated, remains at a constant curvature and has an easily replaceable epithelial cover; all this has to be achieved under conditions in which the fluids bathing it must remain transparent and without any help from the blood through an accessible capillary network. A truly remarkable achievement.

The adaptations which are formed at the limbus are in the arrangement of the blood vessels, the adaption of cellular elements at the limbus to produce multipotential cells, the formation of its own lymphatic system, and modification to the flow of fluids and metabolites. As a consequence of these changes there are quite unusual and in some cases apparently unique changes in the pathological responses of the tissues both to bacterial or viral infections and to the immune responses. These changes are most noticeable in the adjacent cornea but they are also present in the sclera and the coats overlying it, and the vascular tissues beneath.

These articles focus attention on this extraordinary zone and may stimulate others to answer many of the interesting questions that they raise.

P. G. Watson 